\title{
THE DISTRIBUTION OF CUBE-FULL NUMBERS
}

\author{
by P. SHIU
}

(Received 12 March, 1990)

0. Abstract. An elementary derivation of the asymptotic formula for the number of cube-full numbers up to $x$ is given. This derivation is used, together with an estimation of a three dimensional exponential sum, to establish the asymptotic formula for the number of cube-full numbers in the short interval $x<n<x+x^{2 / 3+\theta}$ where $140 / 1123<\theta<1 / 3$.

1. Introduction. Let $k$ be a fixed integer greater than 1. A positive integer $n$ is called a $k$-full number if $p^{k}$ divides $n$ whenever $p$ is a prime divisor of $n$. For $x \geq 1$ we denote by $Q_{k}(x)$ the number of $k$-full numbers not exceeding $x$. The investigation of $k$-full numbers began in 1935 when Erdös and Szekeres [1] gave an asymptotic formula for $Q_{2}(x)$. The best known results concerning the error term $\Delta_{k}(x)$ associated with the asymptotic formula for $Q_{k}(x)$ can be found in the more recent papers of Ivić and Shiu [2] and Krätzel [4].

Concerning the simplest case of the square-full numbers the author [10] (see also [8] and [3] on further development) gave an asymptotic formula for

$$
Q_{2}\left(x+x^{1 / 2+\theta}\right)-Q_{2}(x), \quad 0 \cdot 1526 \leq \theta<\frac{1}{2} ;
$$

the significance of the result is that such an asymptotic formula holds for some $\theta<1 / 6$. It was remarked that the extension of this short interval result to $k \geq 3$ can be obtained if the error term $\Delta_{k}^{*}(x)$ associated with the asymptotic formula for

$$
S_{k}(x)=\sum_{a_{1}^{k} \ldots a_{k}^{2 k-1} \leq x} 1
$$

satisfies

$$
\Delta_{k}^{*}(x) \ll x^{\rho} \quad \text { as } \quad x \rightarrow \infty, \text { for some } \rho<\frac{1}{2 k+2}
$$

This was then known to be the case only for $k=2$, but Krätzel [4] has now established it for $k=3$. Consequently an asymptotic formula for

$$
Q_{3}\left(x+x^{2 / 3+\theta}\right)-Q_{3}(x), \quad \theta_{0}<\theta<\frac{1}{3}
$$

holds with $\theta_{0}=1 / 8$ (see (2.5) and (2.7)), and the method of [10] can be applied to shorten the interval concerned by having $\theta_{0}<1 / 8$; but there is some complication in this which we now explain. As shown in [2], the asymptotic formula for $Q_{k}(x)$ can be obtained by an analytic method via the use of Dirichlet series. However, the case $k=2$ is rather special in that the generating function for $Q_{2}(x)$ is $\zeta(2 s) \zeta(3 s) / \zeta(6 s)$ which is meromorphic in the whole $s$-plane, in contrast to that for $Q_{k}(x), k \geq 3$, which is an infinite product of the Riemann Zeta functions with the line $\sigma=0$ as a natural boundary. Thus, although the elementary derivation of the asymptotic formula for $Q_{2}(x)$ is rather simple we should expect complications when $k \geq 3$. Now the method for the short interval result was adapted from that of Roth [7] for square-free numbers and the method requires an elementary derivation of the asymptotic formula concerned with a "delicate" error term.

Glasgow Math. J. 33 (1991) 287-295. 
In Section 2 we give an elementary derivation of the asymptotic formula for $Q_{3}(x)$ with an error term $O\left(x^{1 / 8}\right)$. Indeed the new proof of the asymptotic formula also adds insight to the arithmetical structure of cube-full numbers which is less transparent in the analytical proof. In Section 3 we state a theorem which implies that there may be no cube-full numbers between successive cubes; this is a generalization of a result in [9], and it explains why we need to consider an interval with length greater than $x^{2 / 3}$ in (1.2). In Section 4 we apply Roth's method to reduce the short interval problem to that of the estimation of the three dimensional exponential sum (4.6). This sum is dealt with in Section 5 using the one dimensional exponent pair method, and our estimate leads to the final result that the asymptotic formula for (1.2) holds when

$$
\frac{140}{1123}<\theta<\frac{1}{3}
$$

in other words we can take $\theta_{0}=140 / 1123=0 \cdot 1246607 \ldots<1 / 8$.

Although (1.1) has yet to be established for $k>3$ it is clear from Section 2 that the corresponding development of the short interval method should present no new difficulties.

2. The asymptotic formula for $Q_{3}(x)$. Since every cube-full integer can be written uniquely as $a^{3} b^{4} c^{5}$, where $b c$ is squarefree, we have

$$
Q_{3}(x)=\sum_{a^{3} b^{4} c^{5} \leq x} \mu^{2}(b c),
$$

where $\mu(n)$ is the Möbius function. The corresponding unweighted sum

$$
S_{3}(x)=\sum_{a^{3} b^{4} c^{5} \leq x} 1
$$

has the asymptotic formula (see [2])

where

$$
S_{3}(x)=A_{3}^{*} x^{1 / 3}+A_{4}^{*} x^{1 / 4}+A_{5}^{*} x^{1 / 5}+\Delta_{3}^{*}(x),
$$

$$
A_{r}^{*}=\sum_{\substack{3 \leq n \leq 5 \\ n \neq r}} \zeta\left(\frac{n}{r}\right), \quad r=3,4,5
$$

and $\Delta_{3}^{*}(x)$ is an error term. Let $\rho^{*}$ be the infimum of the set of all $\rho$ such that $\Delta_{3}^{*}(x) \ll x^{\rho}$ holds as $x \rightarrow \infty$. As we mentioned in the introduction Krätzel [4] proved that

$$
\rho^{*} \leq \frac{22}{177}\left(<\frac{1}{8}\right)
$$

The asymptotic formula for $Q_{3}(x)$ now takes the form

where

$$
Q_{3}(x)=A_{3} x^{1 / 3}+A_{4} x^{1 / 4}+A_{5} x^{1 / 5}+\Delta_{3}(x),
$$

$$
\begin{gathered}
A_{r}=A_{r}^{*} J_{3}\left(\frac{1}{r}\right), \quad r=3,4,5, \\
J_{3}(s)=\prod_{p}\left(1-p^{-8 s}-p^{-9 s}-p^{-10 s}+p^{-13 s}+p^{-14 s}\right) ;
\end{gathered}
$$

see [2] where it is shown that

$$
\Delta_{3}(x) \ll x^{1 / 8}
$$


is a consequence of (2.4). Indeed it is pointed out in [2] that $\Delta_{3}(x)=o\left(x^{1 / 8}\right)$ and that the exponent $1 / 8$ can be reduced if and only if the supremum of the real parts of the zeros of $\zeta(s)$ is less than 1 . As we already remarked in the introduction, the asymptotic formula for (1.2) can be derived trivially from $(2.7)$ when $1 / 8<\theta<1 / 3$.

For the non-trivial range of $\theta<1 / 8$ we first give an elementary proof of the formula (2.5) in which (2.7) holds so that Roth's method can be applied. Crudely speaking the complication in the derivation of (2.5) is due to the fact that the Möbius function is not completely multiplicative. We have, from (2.1),

where

$$
\begin{aligned}
Q_{3}(x) & =\sum_{\substack{a^{3} b^{4} c^{5} \leq x \\
(b, c)=1}} \mu^{2}(b) \mu^{2}(c)=\sum_{\substack{a^{3} b^{4} c^{5} \leq x \\
(b, c)=1}} \mu^{2}(c) \sum_{m d^{2}=b} \mu(d) \\
& =\sum_{\substack{a^{3} b^{4} c^{5} d^{8} \leq x \\
(b d, c)=1}} \mu^{2}(c) \mu(d)=\sum_{d \leq x^{1 / 8}} \mu(d) H\left(\frac{x}{d^{8}}, d\right),
\end{aligned}
$$

$$
H(x, d)=\sum_{\substack{a^{3} b^{4} c^{s} \leq x \\(b d, c)=1}} \mu^{2}(c) ;
$$

and it becomes necessary to relate $H(x, d)$ to $S_{3}(x)$ so that the asymptotic formula (2.3) can be applied. We have

$$
\begin{aligned}
& H(x, d)=\sum_{\substack{a^{3} b^{4} c^{5} \leq x \\
(c, d)=1}} \mu^{2}(c) \sum_{\substack{l|b \\
l| c}} \mu(l) \\
& =\sum_{\substack{a^{3} b^{4} c^{S^{9}} \leq x \\
(c l, d)=1}} \mu^{2}(c l) \mu(l)=\sum_{\substack{a^{3} b^{4} c^{5} l^{9} \leq x \\
(c, l, d)=1}} \mu^{2}(c) \mu(l)
\end{aligned}
$$

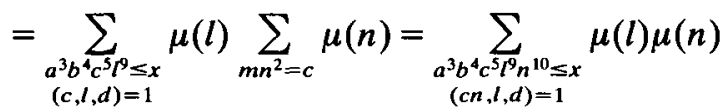

$$
\begin{aligned}
& =\sum_{\substack{p_{n} n^{10} \leq x \\
(l, n, d)=1}} \mu(l) \mu(n) \sum_{a^{3} b^{4} c^{4} \leq x / l^{9} n^{10}} \sum_{\substack{k|c \\
k| d l}} \mu(k) \\
& =\sum_{\substack{3^{3} n^{10} \leq x \\
(l, n, d)=1}} \mu(l) \mu(n) \sum_{k \mid d l} \mu(k) S_{3}\left(\frac{x}{k^{5} l^{9} n^{10}}\right)
\end{aligned}
$$

and substituting (2.3) into here yields

where

$$
H(x, d)=\sum_{r=3}^{5} A_{r}^{*} x^{1 / r} \sum_{\substack{l^{9} n^{10} \leq x \\(l, n, d)=1}} \frac{\mu(l) \mu(n)}{\left(l^{9} n^{10}\right)^{1 / r}} \sum_{k \mid d l} \frac{\mu(k)}{k^{5 / r}}+E(x, d),
$$

$$
E(x, d)=\sum_{\substack{9 \\\left(n^{10} \leq x \\(l, n, d)=1\right.}} \mu(l) \mu(n) \sum_{k \mid d l} \mu(k) \Delta_{3}^{*}\left(\frac{x}{k^{5} l^{9} n^{10}}\right) .
$$

Returning to (2.8) we now have

$$
Q_{3}(x)=\sum_{r=3}^{5} A_{r}^{*} x^{1 / r} Q_{3}(x, r)+\Delta_{3}^{+}(x)
$$


where

and

$$
Q_{3}(x, r)=\sum_{\substack{d^{8} q^{10} 0^{10} \\(d, l, n)=1}} \frac{\mu(d) \mu(l) \mu(n)}{\left(d^{8} l^{9} n^{10}\right)^{1 / r}} \sum_{k \mid d l} \frac{\mu(k)}{k^{5 / r}}
$$

$$
\Delta_{3}^{+}(x)=\sum_{d \leq x^{1 / 8}} \mu(d) E\left(\frac{x}{d^{8}}, d\right) .
$$

We proceed to show that, for $3 \leq r \leq 5$,

$$
Q_{3}(x, r)=J_{3}\left(\frac{1}{r}\right)+O\left(x^{1 / 8-1 / r}\right) .
$$

We shall make use of the identity

$$
\sum_{\substack{n=1 \\(n, d)=1}}^{\infty} f(n)=\prod_{p+d}(1+f(p))=\prod_{p \mid d}(1+f(p))^{-1} \prod_{p}(1+f(p))
$$

for the multiplicative function $f(n)$ with $f\left(p^{j}\right)=0$ for $j \geq 2$ and it will also be convenient to write $v=p^{-1 / r}$. We first evaluate $\lim _{x \rightarrow \infty} Q_{3}(x, r)$, which is given by

$$
\begin{aligned}
\sum_{d=1}^{\infty} & \sum_{\substack{l=1 \\
(l, d)=1}}^{\infty} \frac{\mu(d) \mu(l)}{\left(d^{8} l^{9}\right)^{1 / r}} \sum_{k \mid d l} \frac{\mu(k)}{k^{5 / r}} \sum_{\substack{n=1 \\
(n, d l)=1}}^{\infty} \frac{\mu(n)}{n^{10 / r}} \\
& =\sum_{d=1}^{\infty} \sum_{l=1}^{\infty} \frac{\mu(d) \mu(l)}{\left(d^{8} l^{9}\right)^{1 / r}} \sum_{k \mid d l} \frac{\mu(k)}{k^{5 / r}} \sum_{p \mid d l}\left(1-v^{10}\right)^{-1} \prod_{p}\left(1-v^{10}\right) \\
& =\sum_{d=1}^{\infty} \frac{\mu(d)}{d^{8 / r}} \sum_{k \mid d} \frac{\mu(k)}{k^{5 / r}} \prod_{p \mid d}\left(1-v^{10}\right)^{-1} \prod_{p \mid d}\left\{1-v^{9}\left(1-v^{5}\right)\left(1-v^{10}\right)^{-1}\right\} \prod_{p}\left(1-v^{10}\right) \\
& =\prod_{p}\left\{1-\frac{v^{8}\left(1-v^{5}\right)}{1-v^{10}}\left(1-\frac{v^{9}\left(1-v^{5}\right)}{1-v^{10}}\right)^{-1}\right\}\left\{1-\frac{v^{9}\left(1-v^{5}\right)}{1-v^{10}}\right\}\left(1-v^{10}\right) \\
& =\prod_{p}\left(1-\frac{v^{9}}{1+v^{5}}-\frac{v^{8}}{1+v^{5}}\right)\left(1-v^{10}\right)=\prod_{p}\left(1+v^{5}-v^{8}-v^{9}\right)\left(1+v^{5}\right) \\
& =\prod_{p}\left(1-v^{8}-v^{9}-v^{10}+v^{13}+v^{14}\right) .
\end{aligned}
$$

In view of (2.6) we have proved that $\lim Q_{3}(x, r)=J_{3}(1 / r)$. In order to obtain an error term we note that

$$
\left|\sum_{k \mid d l} \frac{\mu(k)}{k^{5 / r}}\right| \leq \sum_{k=1}^{\infty} \frac{1}{k^{5 / 4}}=\zeta\left(\frac{5}{4}\right) \quad \text { if } \quad r=3,4
$$

and if $r=5$, then the left-hand side is at most 1 . Therefore

$$
\sum_{\substack{d^{8} l^{1} n^{10}>x \\(d, l, n)=1}} \frac{\mu(d) \mu(l) \mu(n)}{\left(d^{8} l^{9} n^{10}\right)^{1 / r}} \sum_{d \mid k l} \frac{\mu(k)}{k^{5 / r}} \ll \sum_{d^{8} l^{1} n^{10}>x}\left(\frac{1}{d^{8} l^{9} n^{10}}\right)^{1 / r} \ll x^{1 / 8-1 / r},
$$

and the formula (2.14) is proved. 
From (2.11) and (2.13) we have

$$
\Delta_{3}^{+}(x)=\sum_{\substack{d^{8} l^{9} n^{10} \leq x \\(d, l, n)=1}} \mu(d) \mu(l) \mu(n) \sum_{k \mid d l} \mu(k) \Delta_{3}^{*}\left(\frac{x}{k^{5} d^{8} l^{9} n^{10}}\right) .
$$

Now let $\rho^{*}<\rho<1 / 8$, and $\epsilon=(1-8 \rho) / 8$. Using Krätzel's estimate $\Delta_{3}^{*}(x) \ll x^{\rho}$ together with

we find that

$$
\sum_{k \mid d l} \frac{1}{k^{5 / 8}} \leq \sum_{k \mid d l} 1 \ll(d l)^{\epsilon}
$$

$$
\Delta_{3}^{+}(x) \ll x^{\rho+\epsilon}=x^{1 / 8} .
$$

The required result (2.7) now follows from (2.12) and (2.14).

3. Cube-full numbers between successive cubes. Before we proceed to establish an interval result for $Q_{3}(x)$ we should mention that there may be no cube-full numbers between successive cubes. In fact we have the following result.

THEOREM 1. Let $f(n)$ denote the number of cube-full integers in the interval $n^{3}<q<(n+1)^{3}$ and let

$$
F_{m}=\{n: f(n)=m\} \quad(m=0,1,2, \ldots) .
$$

Then each $F_{m}$ has positive asymptotic density $d_{m}$ given by

where

$$
d_{m}=\sum_{l=0}^{\infty}(-1)^{l} \frac{(m+l) !}{m ! l !} C_{m+l}
$$

$$
C_{0}=1, \quad C_{r}=\sum_{1<t_{1}<\ldots<r_{r}}^{\prime}\left(\frac{1}{t_{1} \ldots t_{r}}\right)^{1 / 3} ;
$$

here the prime' denotes the summation over integers $t_{i}$ of the form $t_{i}=b^{4} c^{5}$ with $b, c$ coprime and squarefree.

We omit the proof of this theorem which is a straightforward generalization of that for square-full numbers given in [9]. Similarly to the square-full case, if $q_{m}$ is the $m$ th cube-full number we can deduce from the theorem that

$$
\limsup _{m \rightarrow \infty} \frac{q_{m+1}-q_{m}}{3 m^{2}}=\frac{1}{A_{3}}
$$

However, the value of

$$
\liminf _{m \rightarrow \infty}\left(q_{m+1}-q_{m}\right)
$$

has yet to be determined; indeed we cannot even prove that it is finite. This is in contrast to the square-full case where it is easily seen to have the value 1 by appealing to Pell's equation. 
4. A short interval theorem. We shall now be concerned with the number of cube-full numbers in the interval $x<n \leq x+h$. From Theorem 1 we see that this interval may contain no cube-full number at all if $h<x^{2 / 3}$. We therefore write

$$
h=x^{2 / 3+\theta}, \quad 0<\theta<\frac{1}{3} \text {. }
$$

It follows at once from (2.5) and (2.7) that if $1 / 8<\theta<1 / 3$, then

$$
Q_{3}(x+h)-Q_{3}(x) \sim \frac{1}{3} A_{3} x^{\theta} \quad \text { as } \quad x \rightarrow \infty .
$$

We now prove the following result.

THeOREM 2. Let $\theta_{0}$ be the infimum of the set of all $\theta$ such that (4.2) holds. Then

$$
\theta_{0} \leq \frac{2+3 \rho^{*}}{25-48 \rho^{*}}
$$

We remark that the right-hand side of (4.3) is less than $1 / 8$ if and only if $\rho^{*}<1 / 8$, and that the required result (1.3) follows from Krätzel's estimate of $\rho^{*}$ in (2.4). As in [10] we apply Roth's method to investigate $Q_{3}(x+h)-Q_{3}(x)$. Let

$$
\rho^{*}<\rho<\frac{1}{8}, \quad \log x<t<x^{1 / 8} .
$$

From (2.8) we have

$$
Q_{3}(x+h)-Q_{3}(x)=\sum_{\substack{x<a^{3} b^{4} c^{5} d^{8} \leq x+h \\(b d, c)=1}} \mu^{2}(c) \mu(d)
$$

and we divide this sum into $\Sigma_{1}$ and $\Sigma_{2}$ to which the extra conditions of $d \leq t$ and $d>t$ respectively are attached. Thus

$$
\begin{aligned}
\Sigma_{1} & =\sum_{d \leq t} \mu(d) \sum_{\substack{x / d^{8}<a^{3} b^{4} c^{3} \leq(x+h) / d^{8} \\
(b d, c)=1}} \mu^{2}(c) \\
& =\sum_{d \leq t} \mu(d)\left\{H\left(\frac{x+h}{d^{8}}, d\right)-H\left(\frac{x}{d^{8}}, d\right)\right\},
\end{aligned}
$$

where $H(x, d)$ is given by (2.9). From (2.10), (2.11) together with

and

$$
\begin{aligned}
& (x+h)^{1 / 3}-x^{1 / 3}=\frac{1}{3} x^{\theta}+O\left(x^{2 \theta-1 / 3}\right) \\
& (x+h)^{1 / 4}-x^{1 / 4} \ll x^{\theta-1 / 12} \\
& (x+h)^{1 / 5}-x^{1 / 5} \ll x^{\theta-2 / 15}
\end{aligned}
$$

it now follows that

$$
\sum_{d \leq t}\left(\frac{x}{d^{8}}\right)^{\rho} \ll x^{\rho} t^{1-8 \rho}
$$

$$
\Sigma_{1}=\left(\frac{1}{3} A_{3}+O(1)\right) x^{\theta}+O\left(x^{\rho_{t} t^{-8 \rho}}\right)
$$

Using the trivial estimate

$$
\left|\Sigma_{2}\right| \leq \sum_{\substack{x<a^{3} b^{4} c^{5} d^{8} \leq x+h \\ d>t}} 1
$$


and applying the argument in [10] we now find that

$$
\left|Q_{3}(x+h)-Q_{3}(x)-\left(\frac{1}{3} A_{3}+o(1)\right) x^{\theta}\right| \leq\left|U_{3}(x+h, t)-U_{3}(x, t)\right|+O\left(x^{\rho^{1-8 \rho}}\right)
$$

where

$$
U_{3}(x, t)=\sum_{a^{3} b^{4} c^{5} \leq x t^{-8}} \psi\left(\left(\frac{x}{a^{3} b^{4} c^{5}}\right)^{1 / 8}\right)
$$

and, as usual, $\psi(x)=x-[x]-1 / 2$. This three dimensional exponential sum has the trivial estimate

$$
\left|U_{3}(x, t)\right| \leq \sum_{a^{3} b^{4} c^{5} \leq x t^{-8}} 1=S_{3}\left(x t^{-8}\right) \ll x^{1 / 3} t^{-8 / 3} .
$$

However, for our sharper result we need the following non-trivial estimate, the proof of which is given in the next section. As $x \rightarrow \infty$ we have

$$
U_{3}(x, t) \ll x^{1 / 3} t^{-19 / 6} \text { uniformly in } 1 \leq t \leq x^{1 / 14} .
$$

From (4.5) we now have

$$
Q_{3}(x+h)-Q_{3}(x)=\left(\frac{1}{3} A_{3}+o(1)\right) x^{\theta}+O\left(x^{1 / 3} t^{-19 / 6}\right)+O\left(x^{\rho^{1-8 \rho}}\right)
$$

provided that $t \leq x^{1 / 14}$. We set $t=x^{\alpha}$, where $\alpha$ is so chosen that the last two error terms are of the same order; that is

$$
\alpha=\frac{1 / 3-\rho}{19 / 6+1-8 \rho}=\frac{2-6 \rho}{25-48 \rho} .
$$

We remark that this is an admissible choice because, by a classical lattice point theorem of Landau [5], $\rho^{*}$ has the lower bound $1 / 12$ so that, by (4.4), $\alpha<1 / 14$. We now have $x^{1 / 3} t^{-19 / 6}=x^{\rho} t^{1-8 \rho}=x^{\gamma}$, where

and (4.9) becomes

$$
\gamma=\rho+\alpha(1-8 \rho)=\frac{2+3 \rho}{25-48 \rho}
$$

$$
Q_{3}(x+h)-Q_{3}(x)=\left(\frac{1}{3} A_{3}+o(1)\right) x^{\theta}+O\left(x^{\gamma}\right) .
$$

Consequently if $\gamma<\theta$, then the asymptotic formula (4.2) holds. Therefore the number $\theta_{0}$ in the theorem satisfies

$$
\theta_{0} \leq \frac{2+3 \rho}{25-48 \rho}
$$

for every $\rho$ satisfying (4.4). The theorem is established subject to the proof of the estimate (4.8).

We remark that if we use the trivial estimate (4.7) instead of (4.8) then we still have the non-trivial result of

$$
\theta_{0} \leq \frac{1}{11-24 \rho^{*}} \leq \frac{177}{1419}=0 \cdot 1247 \ldots
$$


5. The estimation of $U_{3}(x, t)$. A proper three dimensional method of dealing with the sum $U_{3}(x, t)$ is exceedingly difficult. In fact there is, at the moment, still not a suitable two dimensional procedure similar to the one dimensional exponent pair method that can be applied with the same flexibility and ease to two dimensional sums. We confine ourselves to the one dimensional method by writing the three dimensional sum (4.6) as

$$
U_{3}(x, t)=\sum_{b^{4} c^{5} \leq x t^{-8}} U\left(\frac{x}{b^{4} c^{5}}, t\right)
$$

where

$$
U(x, t)=\sum_{a^{3} \leq x t^{-8}} \psi\left(\left(\frac{x}{a^{3}}\right)^{1 / 8}\right) .
$$

In [6] Richert introduced the notation

$$
R(x ; \alpha, \beta, \gamma)=\sum_{n \leq x^{\alpha}} \psi\left(\frac{x^{\beta}}{n^{\gamma}}\right),
$$

where $\alpha, \beta$ and $\gamma$ are positive constants, and he used the theory of exponent pairs to establish that, as $x \rightarrow \infty$,

$$
R(x ; \alpha, \beta, \gamma) \ll x^{\alpha-1 / 2(\beta-\alpha \gamma)}+x^{\alpha l+(\beta-\alpha \gamma) k / k+1}
$$

whenever $(k, l)$ is an exponent pair satisfying $l>\gamma k$. We set

$$
t=x^{\eta}, \quad \alpha=\frac{1-8 \eta}{3}, \quad \beta=\frac{1}{8}, \quad \gamma=\frac{3}{8},
$$

so that our sum $U(x, t)$ in (5.2) becomes $R(x ; \alpha, \beta, \gamma)$, and that the estimate (5.3) is applicable since every exponent pair satisfies $8 l>3 k$. The first exponent for $x$ in (5.3) is now $(2-19 \eta) / 6$ and, with the exponent pair $\left(\frac{1}{6}, \frac{2}{3}\right)$, the second exponent for $x$ is $(4-29 \eta) / 21$. In other words we have the estimate

$$
U(x, t) \ll x^{1 / 3} t^{-(19 / 6)}+x^{4 / 21} t^{-(29 / 21)} .
$$

Observe that the first error term here dominates over the second error term when $t \leq x^{2 / 25}$ and that the estimate is no better than the trivial estimate of $O\left(\left(x t^{-8}\right)^{1 / 3}\right)$ when $x^{1 / 9} \leq t \leq x^{1 / 8}$. By convexity we may replace the second error term in (5.4) with $\left(x t^{-8}\right)^{\sigma}$, where $\sigma$ satisfies

$$
\frac{1}{3}-\frac{19}{6} \cdot \frac{2}{25}=\sigma\left(1-\frac{8 \times 2}{25}\right)
$$

that is $\sigma=2 / 9$. We therefore have the estimate

$$
U(x, t) \ll x^{1 / 3} t^{-(19 / 6)}+x^{2 / 9} t^{-(16 / 9)}, \text { as } x \rightarrow \infty,
$$


uniformly in $1 \leq t \leq x^{1 / 8}$. Substituting this into our original sum in (5.1), we now have

$$
\begin{aligned}
U_{3}(x, t) & \ll x^{1 / 3} t^{-(19 / 6)}+x^{2 / 9} t^{-(16 / 9)} \sum_{b^{4} c^{5} \leq x t^{-8}}\left(\frac{1}{b^{4} c^{5}}\right)^{2 / 9} \\
& \ll x^{1 / 3} t^{-(19 / 6)}+x^{2 / 9} t^{-(16 / 9)} \sum_{b^{4} \leq x t^{-8}} \frac{1}{b^{8 / 9}} \\
& \ll x^{1 / 3} t^{-(19 / 6)}+x^{2 / 9} t^{-(16 / 9)}\left(x t^{-8}\right)^{1 / 36} \\
& =x^{1 / 3} t^{-(19 / 6)}+x^{1 / 4} t^{-2} .
\end{aligned}
$$

Since the first term on the right-hand side dominates over the second term when $t \leq x^{1 / 14}$ the required estimate (4.8) is established.

\section{REFERENCES}

1. P. Erdốs and G. Sekeres, Über die Anzahl der Abelschen Gruppen gegebeber Ordnung and über ein verwandtes zahlentheoretisches Problem, Acta Sci. Math. (Szeged), 7 (1935), 95-102.

2. A. Ivic and P. Shiu, The distribution of powerful integers, Illinois J. Math. 26 (1982), $576-690$.

3. Jia Chaohua, On square-full members in short intervals, (Chinese) Acta Math. Sinica 30 (1987), 614-621.

4. E. Krätzel, Zweifache Exponentialsummen und dreidiemnsionale Gitterpunktproblem in Elementary and analytic theory of numbers, Warsaw, (1982) Banach Center Publ. 17, 337-369 (PWN Warsaw, 1985).

5. E. Landau, Uber die Anzahl der Gitterpunkte in gewissen Bereichen (II), Nachr. Ges. Wiss. Göttingen (1915), 209-243.

6. H.-E. Richert, Über die Anzahl Abelscher Gruppen gegebener Ordnung (I), Math. Z. 56 (1952), 21-32.

7. K. F. Roth, On the gaps between squarefree numbers, J. London Math. Soc. 26 (1951), 263-268.

8. P. G. Schmidt, Zur Anzahl quadratvollen Zahlen in kurzen Intervallen, Acta Arith. 46 (1986), 159-164.

9. P. Shiu, On the number of squarefull integers between successive squares, Mathematika 27 (1980), 171-178.

10. P. Shiu, On squarefull integers in a short interval, Glasgow Math. J. 25 (1984), 127-134.

Department of Mathematical Sciences

LOUGHBOROUGH UNIVERSTTY

LOUGHBOROUGH

LEICESTERSHIRE LE11 3TU

ENGLAND 